\title{
Defining the Intramedullary Axis of the Distal Tibia Using Computed Tomography
}

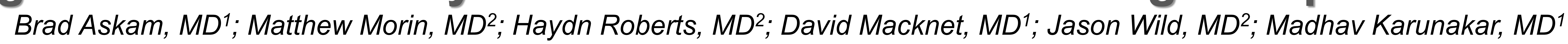

\section{Purpose}

Importance of proper intramedullary nail position within the proximal tibia to prevent deformity in the treatment of proximal tibial shaft fractures has been well described.

With the intramedullary nail constrained within the isthmus of the tibial shaft, proper nail end position in the distal tibia is also important to prevent deformity in the treatment of distal tibial shaft fractures

The purpose of this study was to describe the intramedullary axis of the distal tibia in the coronal and sagittal planes using computed tomography (CT).

\section{Methods}

Analysis of CTs of uninjured tibias was performed.

Coronal images were oriented perpendicular to the trans-malleolar axis (axial view) to simulate a mortise view (Fig 1).

- Tibial isthmus was identified \& plumb line from the center of the isthmus was drawn (coronal) with measurements performed to identify the IM axis (Fig 2).

Sagittal images were oriented perpendicular to the talar body to simulate a lateral view (Fig 1).

- Tibial isthmus was identified (axial view) \& plumb line from the center of the isthmus was drawn (sagittal) with measurements performed to identify the IM axis (Fig 2).

Measurements were converted to a fraction of plafond width and categorized by quadrant.
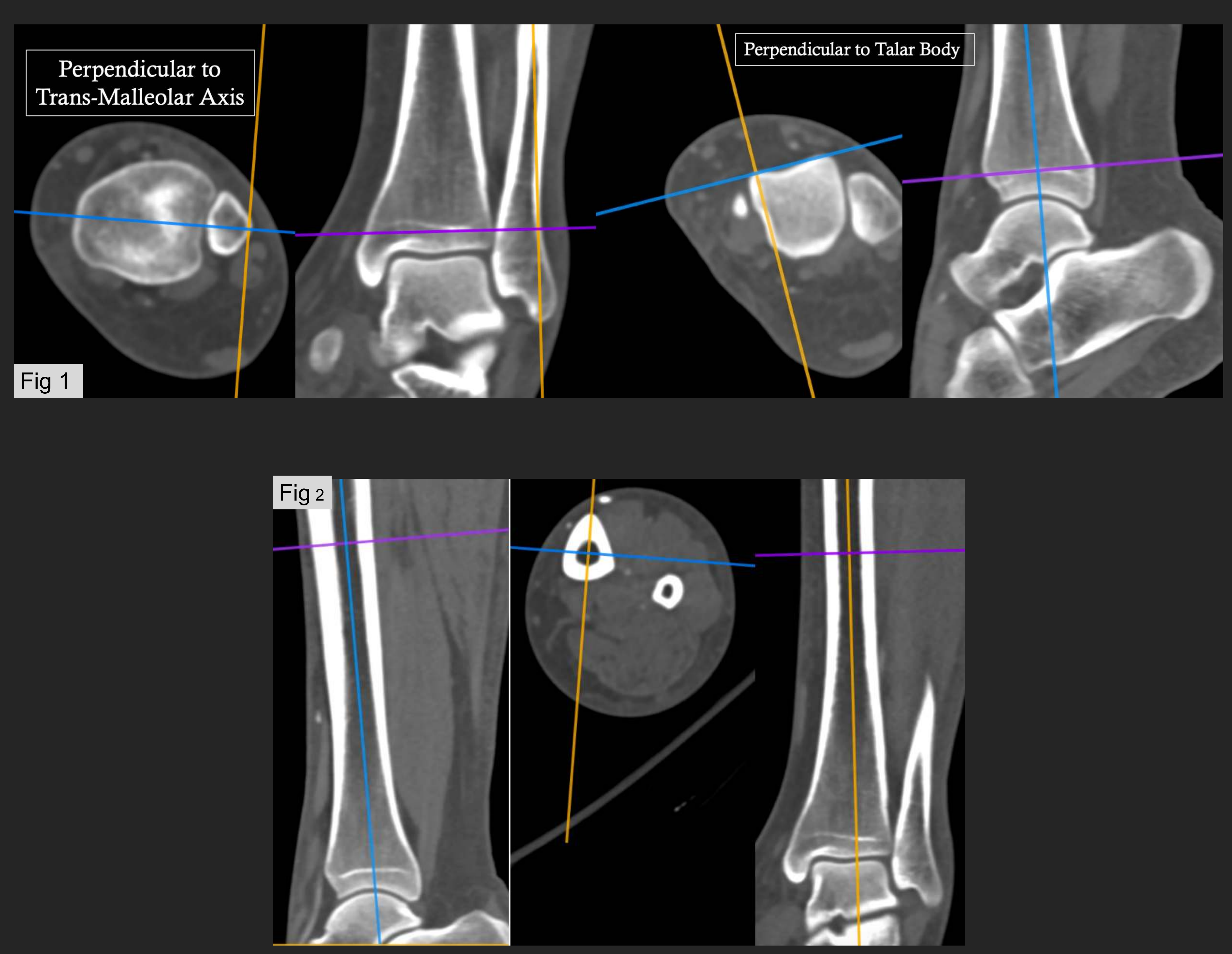

\section{Results}

85 lower extremity CT scans from 82 patients (pts) were analyzed

Coronal Plane - Mean IM axis as a fraction of plafond width (0-1, lateral-medial) was 0.34 (range 0.04-0.71).

Sagittal Plane - Mean IM axis as a fraction of plafond width (0-1, anteriorposterior) was 0.49 (range $0.27-0.74$ ).

The IM axis in the coronal plane was found to be in the lateral quadrant in 10 pts $(12 \%)$; lateral-middle quadrant in 74 pts $(87 \%)$; medial-middle quadrant in 1 pt $(1 \%)$.

The IM axis in the sagittal plane was found to be in the anterior-middle in 52 pts (61\%); and posterior-middle in 33 pts (39\%).
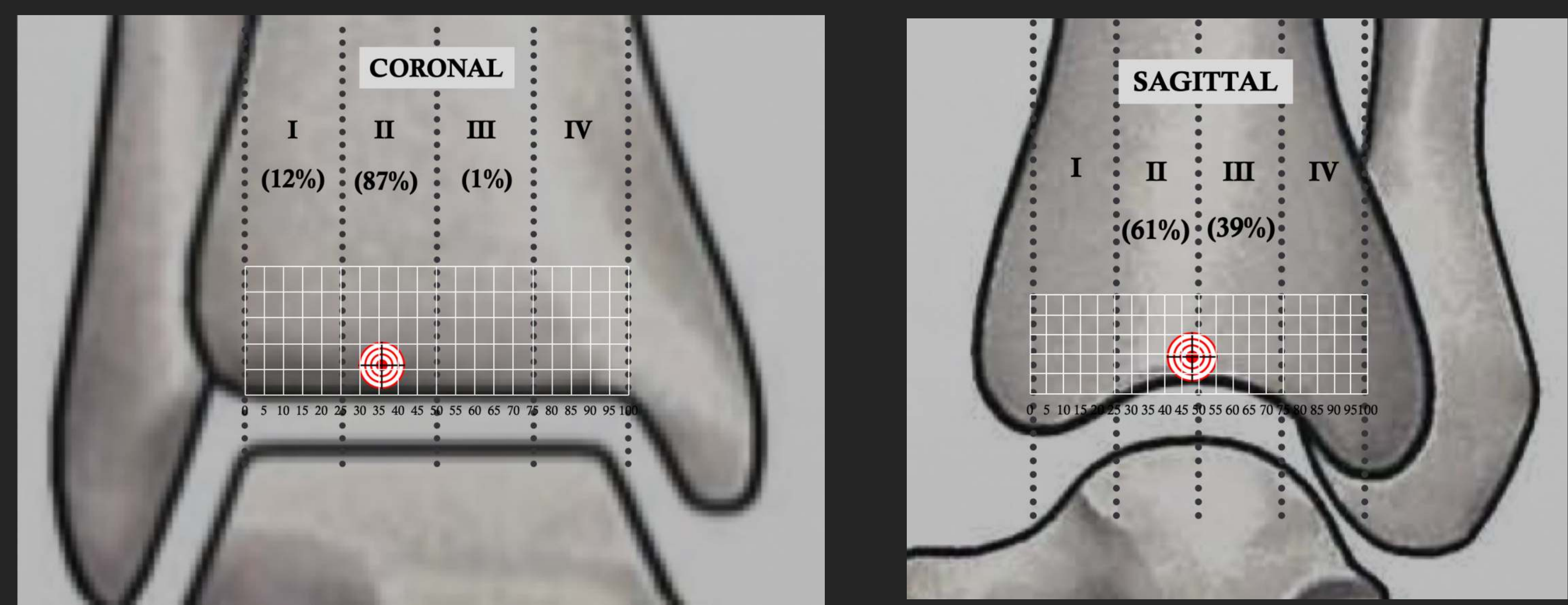

\section{Case Examples}
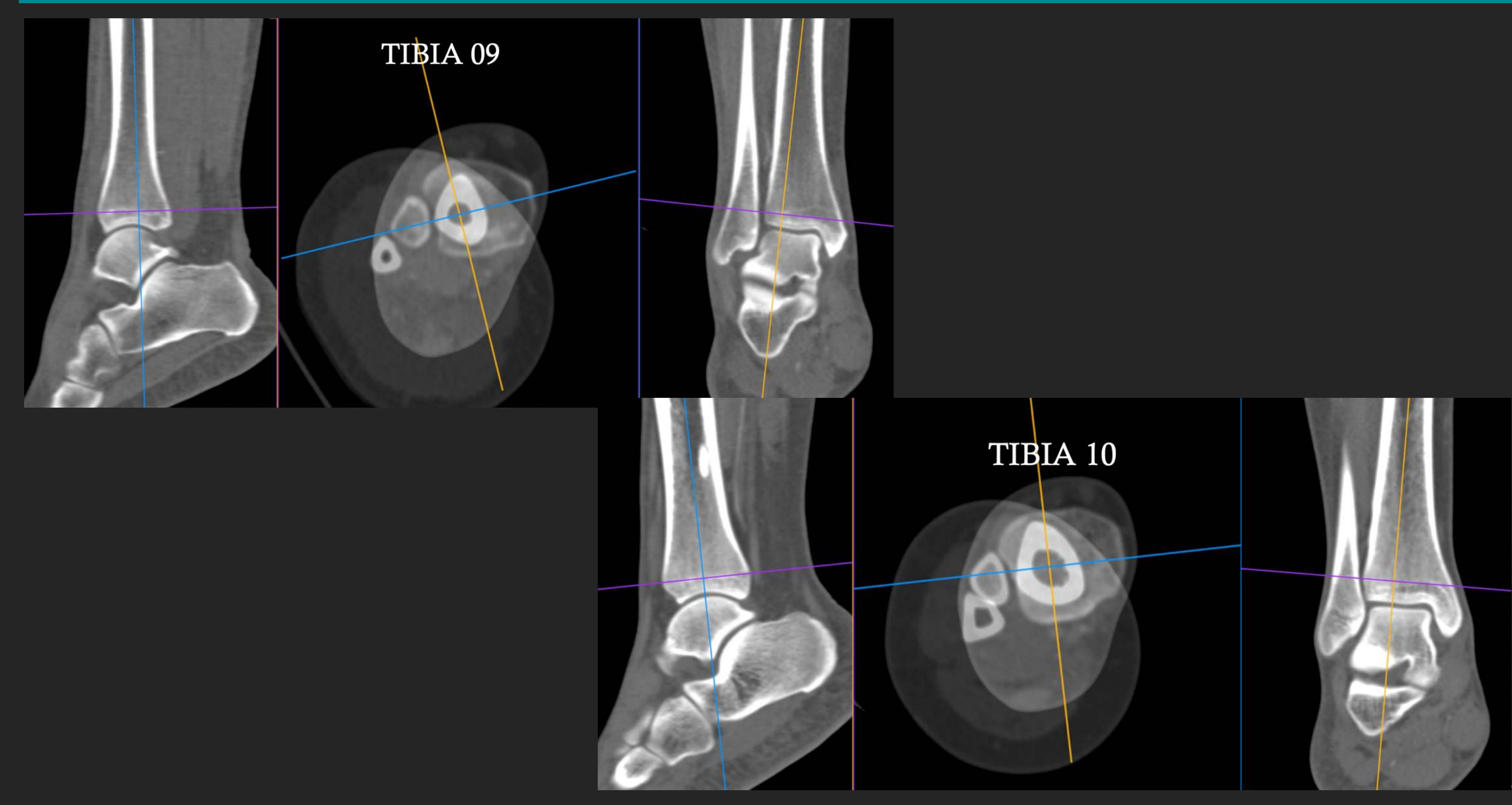

\section{Conclusion}

The IM axis of the distal tibia in the coronal plane most commonly intersects the plafond at a point $1 / 3(0.34)$, the width of the plafond from the incisura.

- $87 \%$ of the measurements fell into the lateral-middle quadrant in the coronal plane.

The IM axis in the sagittal plane fell most commonly in the middle two quadrants and was grouped near "center-center," $(0.49)$

These findings continue to challenge the concept of a "center-center" position for tibial nail end-point, especially in the coronal plane. 